\title{
Retromandibular reduction of medially dislocated condylar process fractures
}

\author{
Gyu Hyeong Lee, Dong Hee Kang, Sang Ah Oh \\ Department of Plastic and Reconstructive Surgery, Dankook University College of Medicine, Cheonan, Korea
}

Background Condylar process fractures account for one-third of all mandibular fractures, and the distal fragment is prone to dislocate to the medial side due to the pulling of the lateral pterygoid muscle. Retromandibular approaches are commonly used, but the intraoperative view becomes limited in medially dislocated fractures. This study summarized a series of cases of retromandibular reduction for medially dislocated condylar process fractures and described our supplementary procedure to realign the dislocated condylar process.

Methods Nine patients with medially dislocated condylar process fractures underwent surgical correction from January 2012 to December 2016. In 6 of them, it was possible to realign the fractures with a conventional retromandibular approach, but for 3 cases of severe dislocation to the middle cranial fossa, a supplementary transoral procedure was carried out. The angle difference between the ramus and condyle, ramus height, and maximal mouth opening (MMO) were evaluated.

Results All 9 cases were restored to the proper anatomical alignment without any major complications, and postoperative images revealed successful union. The angle difference was $8.94^{\circ} \pm 4.11^{\circ}$ preoperatively, and $0.99 \pm 0.49^{\circ}$ at the 6 -month follow-up. The pretreatment ramus height difference was $6.12 \pm 6.09 \mathrm{~mm}$, and the postoperative difference was $0.18 \pm 0.10$ $\mathrm{mm}$. These changes after surgery were statistically significant. The MMO before surgery was $11.44 \pm 3.0 \mathrm{~mm}$, and the postoperative MMO was $37.2 \pm 2.9 \mathrm{~mm}$, reflecting a significant increase after reduction.

Conclusions Retromandibular reduction is a useful method in medially dislocated condylar process fractures, and additional transoral assistance should be considered to realign condylar processes that severely dislocate to the middle cranial fossa.

Keywords Mandibular condyle / Mandibular fractures / Surgical incision
Correspondence: Dong Hee Kang Department of Plastic and Reconstructive Surgery, Dankook University Hospital, Dankook University College of Medicine, 201 Manghyang-ro, Dongnam-gu, Cheonan 31116, Korea Tel: +82-41-550-6477

Fax: +82-41-554-6477

E-mail: dhkcool@daum.net, dhkcool@naver.com

\section{INTRODUCTION}

The condylar region is the most commonly fractured location of the mandible, accounting for $25 \%-35 \%$ of all mandibular fractures $[1,2]$ and condylar process fractures may be classified as intracapsular (involving the condylar head), extracapsular (involving the condylar neck), or subcondylar (involving the low condyle) depending on the height of the fracture. These fractures are also classified by the direction of displacement as medial or lateral [3], and the distal fragments of condylar process fractures are prone to be displaced to the medial side due to the pulling of the lateral pterygoid muscle. In such cases, it is dif- 


\section{Fig. 1. Case of medially dislocated condylar process fracture}

A 52-year-old male patient with a condylar process fracture on the right side. (A) Preoperative coronal computed tomography shows the displaced fracture with medial condylar subluxation. (B) The retromandibular approach offered a limited intraoperative view, and only the proximal cross-section of the distal condylar fragments could be seen by the operator. (C) Preoperative 3-dimensional computed tomography view. (D) Postoperative computed tomography demonstrating good anatomical repositioning of the condylar process.
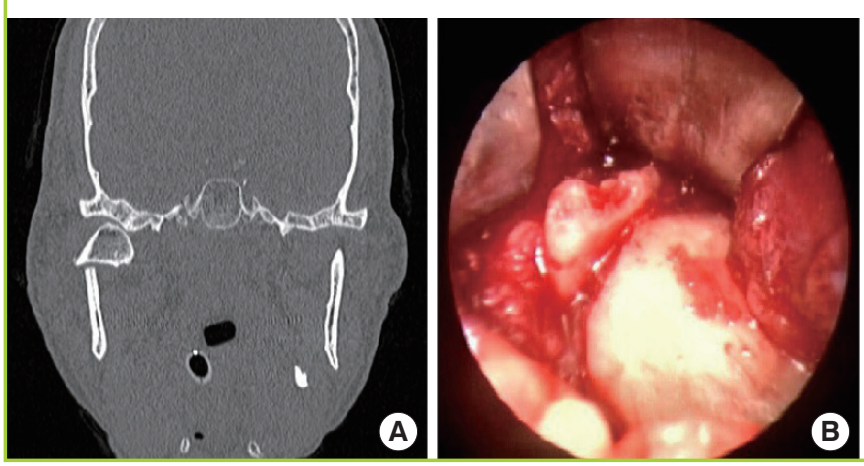

ficult to obtain a good treatment result with conservative treatment due to the persistent pulling of the lateral pterygoid.

Zide and Kent [4] stated that it was an absolute indication for open reduction when the condylar head dislocated to the middle cranial fossa [5], and recent reviews showed that open reduction and internal fixation provided functional outcomes superior to those achieved with conservative treatment in the management of condylar process fractures $[1,6]$. The retromandibular approach provides direct access to the condylar process and a wider space for straightforward fracture management $[6,7]$. However, if the distal condylar segment is displaced inward to the middle cranial fossa, it is hidden behind the proximal segment and the intraoperative view becomes limited. The surgeon can observe only the partial or proximal cross-sectional area of distal condylar fragments through the retromandibular approach (Fig. 1). Therefore, it is difficult to realign the medially displaced condylar fragment, and a large skin incision and more aggressive retraction of the soft tissue may be required to retrieve transversely rotated fracture segments. To overcome these shortcomings, additional procedures are needed to realign the distal condyle fragment for stable fixation.

In this study, we summarized a set of cases of retromandibular reduction performed to treat medially dislocated condylar process fractures, described our additional procedure to realign dislocated condylar processes, and evaluated the functional results and complications associated with this procedure.

\section{METHODS}

\section{Subjects}

Seventy-four patients underwent surgical treatment for mandibular fractures from January 2012 to December 2016, in the Department of Plastic Surgery. The patients provided written

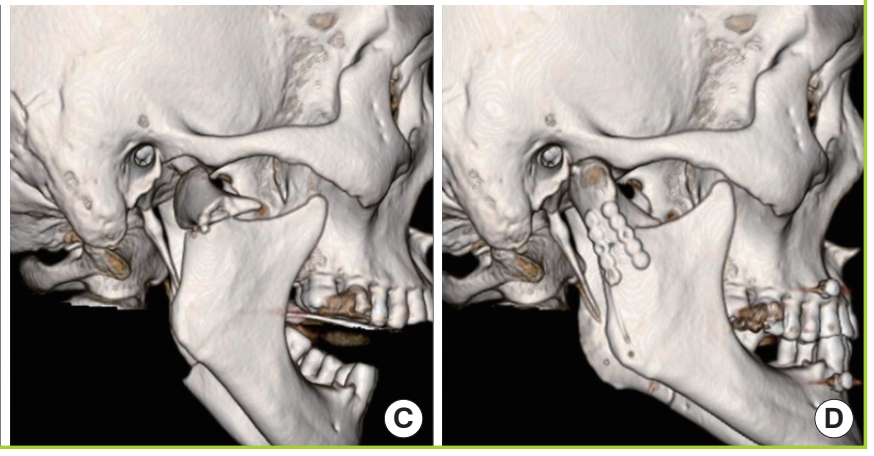

informed consent for the publication and the use of their medical records. Among the 74 patients, 19 were diagnosed with a condylar process fracture. Ten of those patients had a laterally displaced fracture, and they were excluded from this study. Nine patients with medially dislocated condylar fractures were enrolled this study. They consisted of 7 males and 2 females. There were 4 cases of condylar neck fracture and 5 cases of subcondylar fracture. The mean age of the patients was 35.7 years, ranging from 19 to 52 years. The diagnostic protocol included preoperative and postoperative 3-dimensional facial computed tomography (CT) scans and panoramic radiography.

\section{Surgical techniques}

Under general anesthesia, a local anesthetic comprising epinephrine mixed with $2 \%$ lidocaine $(1: 100,000)$ was injected posterior to the mandibular ramus. A 25-mm-long incision was made posterior to the mandibular ramus, and the most proximal point of the incision was just below the earlobe, which runs parallel down to the posterior border of the mandible. When the platysma muscle was shown, blunt dissection was done with a mosquito clip reaching the posterior border of the mandible. This blunt dissection was made behind the parotid gland, which permitted preservation of the integrity of the parotid gland and its capsule. When the facial nerve branch was identified while accessing the condylar process, it was protected with a retractor, but nerve dissection was not performed to avoid nerve damage. The use of a minimal incision with blunt dissection allowed preservation of the facial nerve branches during the operation. Upon exposure of the pterygomasseteric sling, an incision of the periosteum was made along the posterior border. The masseter was then stripped from the ramus and dissected superiorly along the posterior border of the condylar process.

Among the 9 patients, 3 patients with severe medial disloca- 


\section{Fig. 2. Retromandibular approach with transoral assistance}

(A) Dual approach including intraoral and retromandibular incisions. The proximal point of the incision is just below the ear lobe, and runs parallel down to the posterior border of the mandible. (B) Additional support was performed with an elevator via the intraoral approach to retrieve the medially displaced condylar process. (C) The intraoral approach facilitated countertraction against the pull of the lateral pterygoid muscle. (D) The retromandibular incision provided direct visualization and straightforward management of the fractured segments. A 2-plate fixation technique was carried out with a $2.0 \mathrm{~mm}$ dynamic compression plate and a $2.0 \mathrm{~mm}$ mini-adaptation plate to create a stable load on the condylar process.
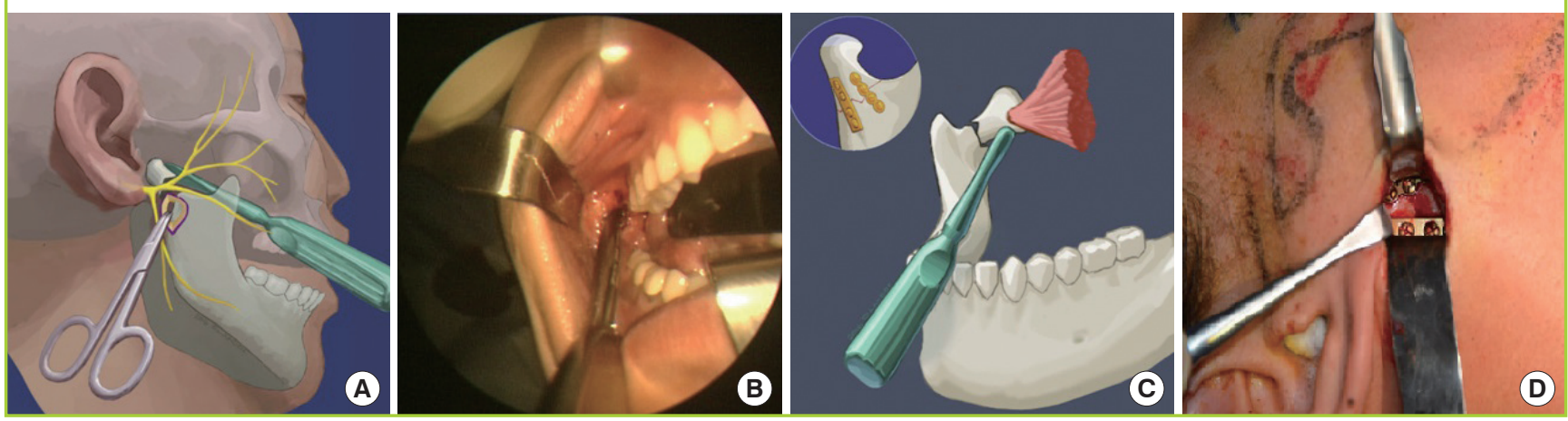

tion of the condylar process underwent a supplementary transoral procedure to reduce the dislocated condylar fragment. An incision was made approximately $5 \mathrm{~mm}$ above the mucogingival junction of the third upper molar, stretching $10 \mathrm{~mm}$ posteriorly. Blunt dissection was carried out with a Gillies elevator until the fractured fragment was reached, and the condylar process was repositioned through the additional transoral approach by pushing the fractured segment outward with the elevator under the view from retromandibular incision. The distal condylar fracture segment was supported by an elevator during the fixation of the fracture from the inside, which facilitated countertraction against the pull of the lateral pterygoid muscle (Fig. 2). Interdental gauze ball packing was used between the upper and lower molar teeth to secure space for fracture restoration in the temporomandibular joint, but no other instrument or traction was used. When a condylar process fracture was combined with other mandibular fractures, condylar process fixation was performed as the top priority. A 2-plate fixation technique was carried out with a $2.0 \mathrm{~mm}$ dynamic compression plate (Synthes, West Chester, PA, USA) or a $2.0 \mathrm{~mm}$ mini-adaptation plate to provide enough strength to withstand the functional load of the condylar process. Reapproximation of the pterygoid masseteric sling and repair of the subcutaneous and cutaneous layers were performed after fracture reduction and plating. A small silastic drain was inserted in the subcutaneous plane to prevent hematoma. Following fracture reduction, a short period of intermaxillary fixation was applied with elastic rubber bands with intermaxillary screws for 2 to 5 days depending on the individual patient's occlusal condition, and a limited range of early mouth opening exercises were started after removing the intermaxillary fixation.

\section{Postoperative evaluation}

Dental occlusion, maximal mouth opening (MMO), and complications were included as postoperative clinical parameters. Articulation and bone healing were evaluated with X-rays and CT scans 6 months after surgical treatment. MMO was measured to assess the range of motion at the day before surgery and after 6 months, and defined as the distance between the upper and lower incisors when the patients opened their mouth as wide as possible.

Panoramic radiography was taken before and 6 months after surgery. The angle between the ramus and condylar head was measured using panoramic radiography to evaluate the degree of sagittal displacement of the fractured condyle. Preoperative and postoperative ramus height were also measured and compared in the panoramic view.

\section{Statistical analysis}

The Wilcoxon signed rank test was used for analysis of the degree of displacement. The significance level was set at a P-value $<0.05$. All analyses were performed with IBM SPSS Statistics for Windows ver. 20.0 (IBM Corp., Armonk, NY, USA).

\section{RESULTS}

Nine patients ( 7 male and 2 female) with medially dislocated mandible condylar process fractures underwent surgical correction (Table 1). The mean age was $35.7 \pm 11.28$ years (range, 1952 years) and there were 5 fractures on the left side and 4 on the right side. The mean follow-up period was $12.7 \pm 10.07$ months (range, 6-36 months). All 9 cases of condylar process fracture were reconstructed to the proper anatomical alignment, and follow-up CT images revealed successful union without any major 
Table 1. Demographic information of the 9 patients included in this study

\begin{tabular}{|c|c|c|c|c|c|c|c|c|}
\hline Patient no. & $\begin{array}{l}\text { Sex/Age } \\
(y r)\end{array}$ & Mechanism & $\begin{array}{c}\text { Condylar } \\
\text { process fracture }\end{array}$ & $\begin{array}{l}\text { Associated } \\
\text { mandibular } \\
\text { fractures }\end{array}$ & $\begin{array}{l}\text { Intraoral } \\
\text { assistance }\end{array}$ & $\begin{array}{l}\text { IMF period } \\
\text { (day) }\end{array}$ & $\begin{array}{l}\text { Follow-up } \\
\quad(\mathrm{mo})\end{array}$ & Complications \\
\hline 1 & Male/35 & Assault & Left subcondyle & Symphysis & Yes & 5 & 12 & \\
\hline 2 & Female/20 & Falling down & Right condylar neck & Left body & No & 4 & 36 & \\
\hline 3 & Female/42 & Falling down & Right condylar neck & No & No & 2 & 6 & \\
\hline 4 & Male/32 & MVA & Right condylar neck & Left angle & Yes & 5 & 6 & \\
\hline 5 & Male/19 & MVA & Left subcondyle & Parasymphysis & No & 2 & 18 & \\
\hline 6 & Male/31 & MVA & Left subcondyle & No & No & 3 & 7 & \\
\hline 7 & Male/43 & MVA & Left condylar neck & Right angle & No & 4 & 18 & Condylar head partial resorption \\
\hline 8 & Male/52 & MVA & Right subcondyle & Left body & Yes & 5 & 6 & \\
\hline 9 & Male/47 & Falling down & Left subcondyle & Symphysis & No & 3 & 6 & \\
\hline
\end{tabular}

Table 2. Mouth opening, ramus height, and angle difference of the condylar process fractures

\begin{tabular}{|c|c|c|c|c|c|}
\hline \multirow{2}{*}{ Variable } & \multicolumn{2}{|c|}{ Pretreatment } & \multicolumn{2}{|c|}{ Postoperative } & \multirow{2}{*}{$\begin{array}{c}\text { P-value } \\
\text { (significance) }\end{array}$} \\
\hline & Mean \pm SD & Median & Mean \pm SD & Median & \\
\hline Maximum mouth opening (mm) & $11.44 \pm 3.00$ & 12 & $37.22 \pm 2.99$ & 37 & $<0.05$ \\
\hline Ramus height difference (mm) & $6.12 \pm 6.09$ & 4.21 & $0.18 \pm 0.10$ & 0.17 & $<0.05$ \\
\hline Condyle/ramus angle difference $\left({ }^{\circ}\right)$ & $8.94 \pm 4.11$ & 8 & $0.99 \pm 0.49$ & 1 & $<0.05$ \\
\hline
\end{tabular}

complications such as malocclusion, temporomandibular joint pain, or paralysis of the marginal mandibular branch. Partial bone resorption in the condylar head was observed in 1 case of condylar neck fracture, but no patients complained of any functional problems.

The degree of displacement of the fractured condyle was assessed by panoramic radiographs. The preoperative angle difference between the condyle and ramus was $8.94^{\circ} \pm 4.11^{\circ}$, and became $0.99^{\circ} \pm 0.49^{\circ}$ at the 6 -month follow-up. There was a statistically significant improvement after surgery. The pretreatment ramus height difference was $6.12 \pm 6.09 \mathrm{~mm}$, and the postoperative difference was $0.18 \pm 0.10 \mathrm{~mm}$. The change in the height difference was likewise statistically significant. The mean MMO before surgery was $11.44 \pm 3.0 \mathrm{~mm}$, and the postoperative MMO was $37.2 \pm 2.9 \mathrm{~mm}$, indicating that it improved significantly greater after surgical reduction (Table 2).

\section{DISCUSSION}

The treatment of condylar process fractures remains controversial among mandibular surgeons, and the conservative management of condylar fractures was previously favored. However, if the distal condylar fragment is displaced to the medial side, it is difficult to obtain good results with conservative treatment due to the persistent pulling of the lateral pterygoid muscle. Zide and Kent [4] suggested that the absolute indications for open reduction should include displacement into the middle cranial fossa, inadequate occlusal restoration by closed reduction, lateral extracapsular displacement of the condyle, and a foreign body at the fracture site. Therefore, it is preferred to perform open reduction when the distal condylar fragment is dislocated to medial side $[1,8,9]$.

Extraoral approaches, which are commonly used in treating condylar process fractures, are subdivided into submandibular, retromandibular, and preauricular approaches. The selection of surgical approach varies depending on the height and position of the fractured segment, but the only criterion for selecting the approach is the distance between the incisions at the level of the fracture. The retromandibular approach was initially presented by Hinds and Girotti [10], and was modified by Koberg and Momma [11]. This approach offers a shorter working distance from the skin incision to the condylar process and permits direct visualization and straightforward management of the fractured segments. Because the entire ramus is easily visible from behind, this technique is useful for procedures involving an area extending from the condylar neck to the base of the ramus itself. This approach is easy to perform and the incision length is limited to $25 \mathrm{~mm}$, such that it leaves aesthetically pleasing scars $[1,11]$.

One of the disadvantages of the retromandibular approach is that it is inadequate for accessing medially displaced condylar process fractures. The superior and inferior head of the lateral 


\section{Fig. 3. Case of successful repositioning of condyalr process with dual approach}

A 31-year-old male patient with a condylar process fracture on the left side. (A) Preoperative 3-dimensional (3D) computed tomography (CT) shows a displaced fracture with medial dislocation of the condylar fragment. (B) Postoperative 3D CT demonstrates good anatomical repositioning of the condyle. (C) Preoperative coronal view. (D) Postoperative coronal CT shows that the fractured subcondyle was restored to the anatomic position.
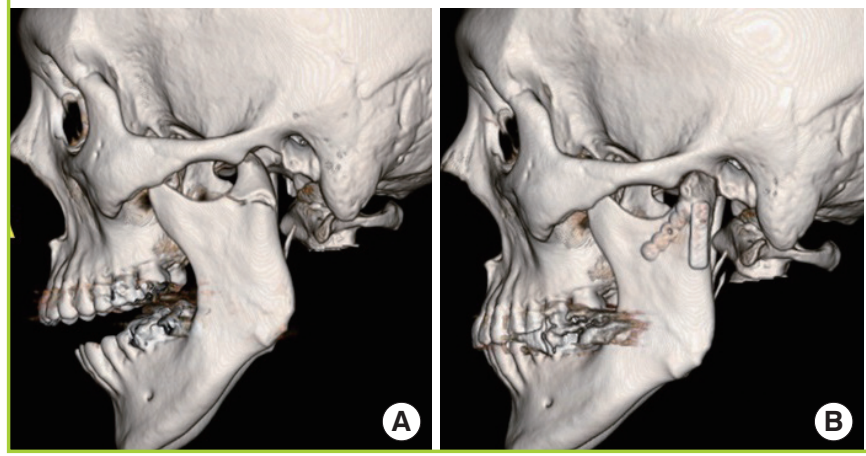

pterygoid inserts onto the neck of the condylar process and fibrous capsule of the temporomandibular joint, and the primary function of lateral pterygoid muscle is to pull the head of the condyle out of the mandibular fossa along the articular eminence to protrude the mandible. Therefore, the distal fragment dislocated from the glenoid fossa is prone to dislocate inside to the middle cranial fossa, due to the pulling of the lateral pterygoid muscle. In this situation, the retromandibular approach offers a limited intraoperative view, as only the partial or proximal cross-section of the rotated condylar fragments can be observed by the surgeon. This makes it difficult for surgeons to perform successful bone realignment through small retromandibular approaches. Instruments such as a curved Freer elevator and Salzburg retractor can be used, but it is difficult to handle the floating distal condylar fragment through the small incision. A large skin incision and more aggressive retraction of the soft tissue are required to realign transversely rotated fracture segments.

In this study, 9 patients were diagnosed with medially dislocated fractures in 19 condylar process fracture cases, and 3 of the fractures were dislocated to the middle cranial fossa, with a severe angle between the distal and proximal fracture segments. The authors performed surgical reduction of medial dislocated condylar process fractures in 9 patients and it was possible to realign the distal condylar fragments using the conventional retromandibular approach in 6 of them. However, an additional transoral procedure was needed in 3 cases with severe dislocation to the middle cranial fossa. An intraoral incision, approximately 10 $\mathrm{mm}$ in length, was made medial to the coronoid process and followed by blunt dissection with a Gillies elevator. The fractured segments were pushed outward, counteracting the force of the lateral pterygoid muscle and the transoral support was maintained during the plate fixation. Our results showed that medially dislocated condylar fractures were reconstructed successfully
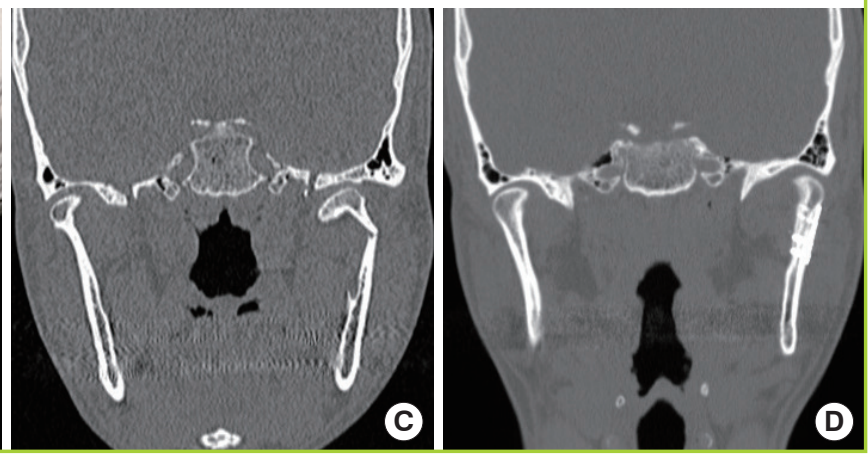

with this transoral assistance, without any complications (Figs. $1,3)$. Therefore, retromandibular condylar process fracture reduction is a useful method for treating condylar process fractures, and the transoral assistance should be considered for the realignment of condylar process fractures that have been severely dislocated into the middle cranial fossa.

\section{CONFLICT OF INTEREST}

No potential conflict of interest relevant to this article was reported.

\section{PATIENT CONSENT}

The patient provided written informed consent for the publication and the use of their images.

\section{REFERENCES}

1. Kang DH. Surgical management of a mandible subcondylar fracture. Arch Plast Surg 2012;39:284-90.

2. Haug RH, Prather J, Indresano AT. An epidemiologic survey of facial fractures and concomitant injuries. J Oral Maxillofac Surg 1990;48:926-32.

3. Bos RR, Ward Booth RP, de Bont LG. Mandibular condyle fractures: a consensus. Br J Oral Maxillofac Surg 1999;37:879.

4. Zide MF, Kent JN. Indications for open reduction of mandibular condyle fractures. J Oral Maxillofac Surg 1983;41:8998.

5. Liu CK, Liu P, Meng FW, et al. The role of the lateral pterygoid muscle in the sagittal fracture of mandibular condyle (SFMC) healing process. Br J Oral Maxillofac Surg 2012; 
50:356-60.

6. Handschel J, Ruggeberg T, Depprich R, et al. Comparison of various approaches for the treatment of fractures of the mandibular condylar process. J Craniomaxillofac Surg 2012; 40:e397-401.

7. Villarreal PM, Monje F, Junquera LM, et al. Mandibular condyle fractures: determinants of treatment and outcome. J Oral Maxillofac Surg 2004;62:155-63.

8. Kim HJ, Roh TS, Tark KC, et al. Outcome of surgical treatment for mandibular condyle fractures. J Korean Soc Plast
Reconstr Surg 2004;31:632-6.

9. Haug RH, Assael LA. Outcomes of open versus closed treatment of mandibular subcondylar fractures. J Oral Maxillofac Surg 2001;59:370-5.

10. Hinds EC, Girotti WJ. Vertical subcondylar osteotomy: a reappraisal. Oral Surg Oral Med Oral Pathol 1967;24:164-70.

11. Koberg WR, Momma WG. Treatment of fractures of the articular process by functional stable osteosynthesis using miniaturized dynamic compression plates. Int J Oral Surg $1978 ; 7: 256-62$ 